OPEN

SUBJECT AREAS:

RESTRICTION FRAGMENT LENGTH POLYMORPHISM

BIODIVERSITY

ANTIGEN PRESENTATION

ZOOLOGY

Received

30 July 2012

Accepted

23 August 2012

Published

20 September 2012

Correspondence and requests for materials should be addressed to

W.A. (wa100@

hermes.cam.ac.uk)

\section{MHC genotype and near-deterministic mortality in grey seals}

\author{
M. de Assunção-Franco' , J. I. Hoffman ${ }^{2}$, J. Harwood ${ }^{3}$ \& W. Amos'
}

\begin{abstract}
'Department of Zoology, University of Cambridge, Downing Street, Cambridge, CB2 3EJ, UK, ${ }^{2}$ Department of Animal Behaviour, University of Bielefeld, Postfach 100131, 33501 Bielefeld, Germany, ${ }^{3}$ Sea Mammal Research Unit, Scottish Oceans Institute, University of St Andrews, St Andrews, Fife, KY16 8LB, UK.
\end{abstract}

The Major Histocompatability Complex (MHC) is one of the best known and best characterised components of the immune system, yet its functions remain somewhat enigmatic, including both anti-pathogen activity and kin recognition. To explore the importance of the MHC relative to literally hundreds of other components of the immune system, we compared MHC genotype frequencies between pups and adults in the grey seal (Halichoerus grypus), one of many marine mammals that exhibit low allelic diversity. We find that one allele is strongly associated with pup survival, pups being more likely to be found dead if they lack it, while total allele number is a remarkably strong predictor of survivorship to adulthood. We estimate that approximately $70 \%$ of mortality can be attributed to the MHC. Our study therefore shows that low MHC allele diversity belies its critical role in determining whether a weaned pup negotiates disease to become a breeding adult.

. he Major Histocompatibility Complex (MHC) is often heralded as a key genetic component of the vertebrate immune system and both total MHC allele number and individual MHC alleles have been implicated in individual survival in natural populations ${ }^{1}$. A remarkable example involves the Seychelles warbler, where early mortality of birds who carry a particular MHC allele creates a five-fold lower survival rate at one point in the survivorship curves, compared with birds who lack this allele ${ }^{2}$. Although the sample size is modest, this difference is statistically significant, suggesting an appreciable role for the MHC in individual survival.

Despite the warbler and other studies, the influence of the MHC on survival in most species seems open to question. On the one hand, the immune system contains many other components, raising the question of what these literally hundreds of genes are for if the MHC on its own is so important. On the other hand, the MHC itself is involved in other functions including olfactory detection of relatedness and mate choice, and MHC allele diversity varies hugely from species to species ${ }^{3}$. Does such variation reflect varying levels of importance that depend critically on a species' ecology, or a varying spectrum of challenges that require more or fewer alleles to provide an effective challenge?

Among species with low MHC diversity appear to be most marine mammals. At face value, this might seem strange since, with few natural predators, disease is likely to play a larger than average role in mortality. An alternative view is that the marine environment might present an unusually difficult set of obstacles to many pathogens, with the size of the oceans, the salt water and the high mobility of most species combining to make most forms of transmission between hosts difficult, at least at times other than when seals, for example, come ashore in dense colonies to breed. To explore this apparent contradiction, we studied the relationship between MHC genotype and mortality in the grey seal (Halichoerus grypus), breeding on the Isle of May, UK.

\section{Results}

We quantified variability at the MHC-DQB locus in a sample of 284 dead pups, 144 live pups and 109 adults (75 females, 34 males) using allele-specific PCR targeting five alleles uncovered in previous studies ${ }^{5}$. The DQB locus is thought to be duplicated since individuals carry up to a maximum of four alleles each, and never all five. As a control, we also analysed existing data from a large sample of adults and pups from the same site, genotyped for a panel of nine presumed neutral microsatellites ${ }^{6}$.

General linear models (GLMs) were fitted with predictor variables presence/absence of each allele, total number of alleles present and the interaction term. The response variable was animal class, fitted in two ways: dead versus live pups (binary) and pups versus adults (binary). Our results are summarised in Table 1 and reveal that, for dead versus live pups, the presence of allele 5 is a strong predictor while total allele number is flagged only as interactions with alleles 3 and 5. In contrast, comparing pups and adults, allele number alone is the key 
Table 1 | Results of Generalized Linear Models (GLMs) of grey seal (Halichoerus grypus) survival. Note that coefficients for interaction terms are omitted since they cannot properly be interpreted in tabular form. $\mathrm{df}$, degrees of freedom. NS not significant.

(a) Dead versus live pups ( $n=428$ )

\begin{tabular}{lcccc}
\hline Term & Estimate & $\dagger$ & df & $P$ \\
\hline Allele 1 & 0.25 & 1.75 & 1 & NS \\
Allele 2 & 0.001 & 0.03 & 1 & NS \\
Allele 3 & -0.08 & 1.19 & 1 & NS \\
Allele 4 & -0.08 & 1.00 & 1 & NS \\
Allele 5 & 0.16 & 3.55 & 1 & 0.0004 \\
Number of alleles & 0.05 & 1.73 & 1 & NS \\
Allele 1: number of alleles interaction & NA & NA & 1 & NS \\
Allele 2: number of alleles interaction & NA & NA & 1 & NS \\
Allele 3: number of alleles interaction & NA & NA & 1 & 0.03 \\
Allele 4: number of alleles interaction & NA & NA & 1 & NS \\
Allele 5: number of alleles interaction & NA & NA & 1 & 0.014 \\
\hline & & & &
\end{tabular}

(b) Pups versus adults ( $n=537$ )

\begin{tabular}{lcccc}
\hline Term & Estimate & $\dagger$ & df & $P$ \\
\hline Allele 1 & 0.21 & 1.69 & 1 & 0.09 \\
Allele 2 & 0.07 & 2.04 & 1 & 0.04 \\
Allele 3 & 0.05 & 0.89 & 1 & 0.38 \\
Allele 4 & 0.07 & 1.25 & 1 & 0.21 \\
Allele 5 & 0.16 & 4.68 & 1 & $3.71 \times 10^{-6}$ \\
Number of alleles & 0.12 & 5.42 & 1 & $8.86 \times 10^{-8}$ \\
Allele 1: number of alleles interaction & NA & NA & 1 & NS \\
Allele 2: number of alleles interaction & NA & NA & 1 & NS \\
Allele 3: number of alleles interaction & NA & NA & 1 & NS \\
Allele 4: number of alleles interaction & NA & NA & 1 & NS \\
Allele 5: number of alleles interaction & NA & NA & 1 & 0.06 \\
\hline
\end{tabular}

predictor. Although allele 5 appears also to predict survival to adulthood, this is almost entirely due to a correlation between possession of allele 5 and allele number. At presumed neutral microsatellite loci, multilocus heterozygosity does not differ significantly between pups and adults, indicating that the differences seen at the MHC are not simply reflecting genomewide patterns. As a control, we also asked whether heterozygosity differed between pups and adults at nine presumed neutral microsatellite loci, using a larger historical dataset $^{6}$. Heterozygosity was quantified as internal relatedness (IR), a measure that weights homozygosity by the frequencies of the alleles carried, with negative values indicating higher heterozygosity. In this sample set, pups (mean IR $=-0.011, \mathrm{n}=854$ ), adult females (mean $\mathrm{IR}=-0.0068, \mathrm{n}=352)$ and adult males (mean IR $=0.0019, \mathrm{n}=$ 223) showed no evidence of variation in heterozygosity (ANOVA, $\mathrm{F}[2,1426]=0.49, \mathrm{NS})$.

Individuals carrying only one MHC allele are almost invariably pups while those with four alleles are mainly adults. This allows estimation of the likely mortality that can be attributable to the number of alleles an individual carries. Assuming that having four alleles confers $100 \%$ survival, those with one allele would on average have to suffer $91 \%$ mortality for their frequency in the population to drop from $18 \%$ in pups to the equivalent of $1.6 \%$ in adults (calculated as raw percentage/proportional increase in frequency of adults who carry four alleles, $=5.5 \% / 3.5)$, and the total required mortality would be $71.5 \%$ (Figure 1). Additional non-MHC-attributable mortality would reduce this differential but increase total mortality. In reality, tagging studies suggest a total pup to adult mortality rate of $60-80 \%$, implying that $62.5-100 \%$ of all deaths are related to $\mathrm{MHC}$ allele number.

\section{Discussion}

Here we explored the relationship between mortality and MHC genotype by comparing genotype frequencies in dead and live pups

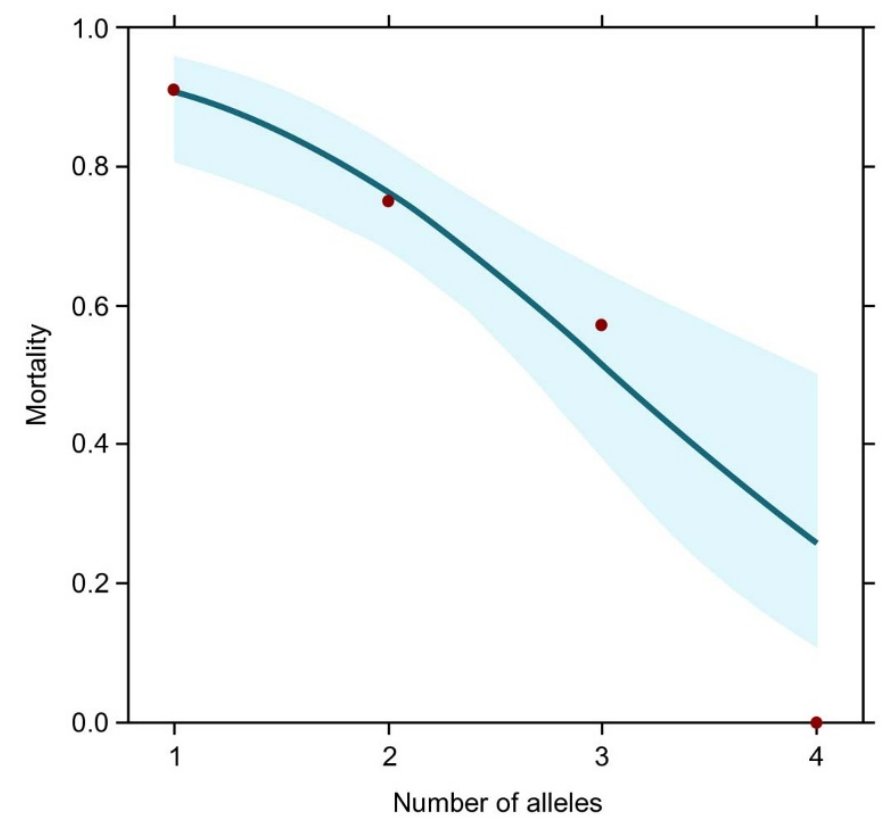

Figure $1 \mid$ Relationship between grey seal (Halichoerus grypus) mortality and MHC allele number. The line shows the fitted values (with $95 \%$ confidence intervals) of the GLM, assuming mortality is 0 for individuals with 4 alleles.

with those in adult seals. We find that one allele is a strong predictor of whether a pup is found dead or alive, while total MHC allele number exerts a strong influence on whether pups survive to enter the breeding population. Overall, the MHC appears to play a dominant role in dictating which animals are most likely to die and at what age.

Our results provide a remarkable example of how mortality in a large mammal can be highly non-random, instead being predictable from each individual's MHC genotype. The idea that upwards of 70\% of grey seal mortality can be attributable to an individual's MHC genotype is truly remarkable and recalls the patterns reported for the Seychelles warbler ${ }^{2}$, an endangered passerine bird. However, the situation with the grey seals is arguably more extreme, in that the confidence intervals around the maximum difference between two survival curves of birds who carry one of several MHC alleles in a sample size of around 100 individuals are likely to be quite large. Moreover, our analysis is so far rather crude, in that we do not have adult age, measures of season quality or other parameters that the warbler study was able to fit. Regardless, it is interesting that these strong effects are both found in species with rather few MHC alleles who largely lack predators, suggesting that in such species disease plays a pivotal role in survival.

Our data imply contrasting mechanisms responsible for protecting pups against early mortality and for allowing successful recruitment into the breeding adult population. However, the exact mechanism remains unclear. One possibility is that grey seals face a small number of threats from key pathogens that impact on different parts of the life cycle, and each of these is more likely to be overcome by possessing a different MHC allele. In this sense, pup mortality would be merely one of several challenges. Alternatively, after weaning there may be a range of pathogens, each of which tends to impact on a seal's physical condition, such that individuals with more MHC alleles are generally more healthy. We tend to favour a model based on one-to-one allele-pathogen interactions because the less-specific model would seem always to select for higher allelic diversity, which, based on other species, seems relatively easy to generate. 
The low MHC allele diversity carried by grey seals rather belies the large effect size we report. A previous study has indicated a role for genome-wide heterozygosity in pre-weaning pup survival ${ }^{8}$, though compared with the MHC effects reported here, heterozygosity seems to exert a much smaller effect in pups and there is little evidence of an impact on pup to adult survival. Whether other components of the immune system are important remains to be seen, though our data suggest, compared with other species, that the MHC is relatively dominant in the grey seal. At the same time, low allele diversity suggests that the marine environment may limit the range of pathogens being faced ${ }^{4}$, making it relatively more important to carry the right alleles.

Previous studies have also highlighted unusual patterns of mate choice in grey seals. While behavioural observations suggest a classical polygynous mating system ${ }^{9}$, some individual females reveal evidence of mate fidelity ${ }^{10}$. At the same time, females who do not show mate fidelity appear to choose mates who are less genetically similar compared with previous partners ${ }^{11}$. Both strategies should help reduce the risk of inbreeding. However, if $\mathrm{MHC}$ allele number is so important in terms of survival, it would seem likely that mate choice in favour of MHC dissimilar partners would be present. This is clearly an interesting avenue for future research.

In conclusion, our results provide a strong support for the notion that the MHC gene complex can exert a major influence on survival. In line with studies showing that animals use olfactory clues associated with MHC genotype as a basis for mate choice ${ }^{12,13}$, it seems that female grey seals have much to gain from choosing MHC different males, since these will father pups who are more likely to survive to adulthood by dint of their increased MHC heterozygosity and greater chance of carrying allele 5 .

\section{Methods}

Tissue sampling and genetic analysis. Tissue samples were collected as part of ongoing studies using methods detailed elsewhere ${ }^{6}$. For this study we sampled 537 individuals comprising dead pups $(\mathrm{n}=284)$, live pups $(\mathrm{n}=144)$, adult females $(\mathrm{n}=75)$ and adult males $(n=34)$. DNA extraction and PCR amplification was performed as described in detail by Cammen et al. ${ }^{5}$. For each sample, DQB PCR amplification was conducted twice followed by digestion with restriction enzymes: one with a combinations of three restriction enzymes (HhaI, NlaIII and MboI) and the duplicate with AvaII separately. The three enzymes combined cut the five MHC sequences (Hagr-DQB*01-05) into unique fragment combinations. The digestion with AvaII confirmed the presence of allele Hagr-DQB*03. Overnight restriction digestion was carried out in a $15 \mu \mathrm{l}$ total volume using 1 unit of each enzyme and a final concentration of $0.9 \times$ NEB buffer 4 . Digested samples were ran on $6 \%$ Polyacrylamide gels and visualised by autoradiography. Resulting gels were scored manually by two experienced observers (MF and JIH).

Statistical analysis. Data analyses were conducted using general linear models (GLMs) within $\mathrm{R}^{14}$. The following models were fitted:

(i) state $\sim$ num

(ii) state $\sim$ allele

(iii) state $\sim$ num $*$ allele

where state $=$ either (i) dead versus live pups; or (ii) all pups versus adults (both modeled using a binomial error structure with dead $=0$, live $=1$ and $\mathrm{pup}=0$, adult $=1)$, and num $=$ number of alleles present $(1$ through 4$)$; and allele $=$ a binary variable $(0=$ absent, $1=$ present $)$ fitted separately for each of the five different alleles. For dead pups versus live pups, where number of alleles is non-significant, significance of each of the five class (iii) models was assessed using ANOVA to test the change in deviance between this model and the model class (ii) in which the allele was fitted alone. For pups versus adults, significance of each of the five class (iii) models was assessed using ANOVA to test the change in deviance between this model and model (i).

1. Sommer, $\mathrm{S}$. The importance of immune gene variability (MHC) in evolutionary ecology and conservation. Front. Zool. 2, 16 (2005).

2. Brouwer, L., Barr, I., Van de Pol, M., Burke, T., Komdeur, J. \& Richardson, D. S. MHC-dependent survival in a wild population: evidence for hidden genetic benefits gained through extra-pair fertilizations. Mol. Ecol. 19, 3444-3455 (2010).

3. Piertney, S. B. \& Oliver, M. K. The evolutionary ecology of the major histocompatibility complex (MHC). Heredity 96, 7-21 (2006).

4. Slade, R. W. Limited MHC polymorphism in the southern elephant seal: implications for MHC evolution and marine mammal population biology. Proc $R$ Soc Lond B Biol Sci 249, 163-171 (1992).

5. Cammen, K., Hoffman, J. I., Knapp, L. A., Harwood, J. \& Amos, W. Geographic variation of the major histocompatibility complex in Eastern Atlantic grey seals (Halichoerus grypus). Mol. Ecol. 20, 740-752 (2011).

6. Worthington Wilmer, J., Allen, P. J., Pomeroy, P. P., Twiss, S. D. \& Amos W. Where have all the fathers gone? An extensive microsatellite analysis of paternity in the grey seal (Halichoerus grypus). Mol. Ecol. 8, 1417-1429 (1999).

7. Pomeroy, P., Smout, S., Moss, S., Twiss, S. \& King, R. Low and delayed recruitment at two grey seal breeding colonies in the UK. J. Northwest Atl. Fish. Sci. 42, 125-133 (2010)

8. Bean, K., Amos, W., Pomeroy, P. P., Twiss, S. D., Coulson, T. N. \& Boyd, I. L. Patterns of parental relatedness and pup survival in the grey seal (Halichoerus grypus). Mol. Ecol. 13, 2365-2370 (2004).

9. Anderson, S. S., Burton, R. W. \& Summers, C. F. Behaviour of grey seals (Halichoerus grypus) during a breeding season at North Rona. J. Zool. 177, 179-195 (1975).

10. Amos, W., Twiss, S. D., Pomeroy, P. P. \& Anderson, S. S. Evidence for Mate Fidelity in the Gray Seal. Science 268, 1897-1899 (1995).

11. Amos, W., Worthington Wilmer, J. \& Kokko, H. Do female grey seals select genetically diverse mates? Anim. Behav. 62, 157-164 (2001).

12. Roberts, S. C., Gosling, L. M., Carter, V. \& Petrie, M. MHC-correlated odour preferences in humans and the use of oral contraceptives. Proc R Soc Lond B Biol Sci 275, 2715-2722 (2008).

13. Wedekind, C. \& Furi, S. Body odour preferences in men and women: Do they aim for specific MHC combinations or simply heterozygosity? Proc R Soc Lond B Biol Sci 264, 1471-1479 (1997).

14. R Development Team. R: a language and environment for statistical computing. Vienna (Austria): R Foundation for Statistical Computing (2005).

\section{Acknowledgments}

We thank Kyi Bean, Don Bowen, Ailsa Hall, Rob Harris, Bernie McConnell, Penny Allen, Paddy Pomeroy, Sean Twiss and Dorete Bloch for assistance with collecting tissue samples. Maria de Assunção-Franco was funded by the Fundação para a Ciêcie e a Tecnologia (FCT). We also acknowledge support for the publication fee by the Deutsche Forschungsgemeinschaft and the Open Access Publication Funds of Bielefeld University.

\section{Author contributions}

All authors conceived of the project and approved the manuscript. JH was responsible for collecting the tissue samples. MAF and JIH conducted the laboratory work. WA, MAF and $\mathrm{JIH}$ analysed the data and wrote the main manuscript text.

\section{Additional information}

Competing financial interests: The authors declare no competing financial interests

License: This work is licensed under a Creative Commons

Attribution-NonCommercial-NoDerivative Works 3.0 Unported License. To view a copy of this license, visit http://creativecommons.org/licenses/by-nc-nd/3.0/

How to cite this article: de Assunção-Franco, M., Hoffman, J.I., Harwood, J. \& Amos, W. MHC genotype and near-deterministic mortality in grey seals. Sci. Rep. 2, 659; DOI:10.1038/srep00659 (2012). 